DOI: $10.2478 /$ lpts-2021-0040

\title{
RESEARCH OF KINEMATIC STEPPING MECHANISM
}

\author{
I. Grinevich*, V. Nikishin, G. Springis \\ Riga Technical University, Daugavpils Affiliation, \\ 90 Smilsu Str., Daugavpils, LV-5410, LATVIA \\ *e-mail: Ivans.Grinevics@rtu.Iv
}

In this paper, a kinematic analysis of Theo Jansen's stepping mechanism has been carried out and an algorithm for finding the output link trajectory from the given dimensions of the stepping mechanism elements, implemented by Mathcad program, has been developed. It is possible to output characterising parameters of all intermediate links with any number of intermediate links of a step cycle of the mechanism. The dimensions of the mechanism elements have been selected so that they provide the optimal smooth trajectory of the stepping point, minimising the mechanism oscillations in the vertical plane. A comparison of the trajectories of the foot in this study with the classical trajectory of Theo Jansen and the trajectory from article [7] has been provided. A minimum swing of the oscillation of the centre of mechanism masses in the vertical plane has been selected as an optimality criterion, combined with the maximum smoothness of the trajectory, provided that maximization of the step height is not required.

Keywords: Kinematic analysis, step trajectory, Theo Jansen mechanism.

\section{INTRODUCTION}

Robotics is one of the fastest growing areas of modern science. A topical direction in this area is the development and study of stepping mechanisms. Theo Jansen's [1], [2] stepping mechanism, simulating the gait of a living organism, is widely-known. Theo Jansen's "beach animals" created on this principle from plastic pipes are set in motion by the force of the wind. In [3], an overview of the work on the applications of the Jansen mechanism in various fields is given. It was stated that the majority of developments were at the research stage. In [4], a detailed study of the Jansen mecha- 
nism was carried out, the trajectory of the stepping point was determined, and a modification of the basic mechanism was proposed in order to improve its characteristics. In [5], a method for increasing the maneuverability through a mechanism with 5 links implementing a step height change was proposed. To find the trajectory of the proposed mechanism and the Jansen mechanism, an analytical method was applied using complex number forms. The problem of stability of stepping mechanisms was investigated in [6]. In [7], a structural analysis of the Jansen mechanism was carried out and the trajectory of the output link was determined from 12 intermediate positions.

\section{THEORY}

The scheme of the stepping mechanism (Theo Jansen's mechanism) is shown in Fig. 1. It consists of two stiff triangles $\mathrm{AON}, \mathrm{FBT}$ and rods $\mathrm{KA}=\mathrm{a}, \mathrm{KB}=\mathrm{z}$, $\mathrm{NF}=\mathrm{g}, \mathrm{OB}=1, \mathrm{O}_{1} \mathrm{~K}=\mathrm{r}$. Points $\mathrm{A}, \mathrm{O}, \mathrm{N}, \mathrm{B}$, $\mathrm{F}, \mathrm{K}, \mathrm{O}_{1}$ are hinges, with $\mathrm{O}$ and $\mathrm{O}_{1}$ being fixed firmly. By rotating the rod $\mathrm{O}_{1} \mathrm{~K}(\mathrm{r})$ against the clock, the mechanism is set in motion. The step trajectory is implemented by vertex $\mathrm{T}$ of triangle FBT. Let us denote the sides of the triangles as $\mathrm{AN}=\mathrm{w}, \mathrm{OA}=\mathrm{v}$, $\mathrm{ON}=\mathrm{u}, \mathrm{FB}=\mathrm{f}, \mathrm{BT}=\mathrm{p}, \mathrm{FT}=\mathrm{s}, \angle \mathrm{AON}=\alpha$, $\angle \mathrm{FBT}=\beta$, distance $\mathrm{OO}_{1}=\mathrm{d}$. It is necessary to select numerical values for parameters $r$, a, z, d, g, l, v, u, w, f, p, s, which provide the optimal trajectory of point $\mathrm{T}$. We introduce a rectangular coordinate system $\mathrm{xOy}$ (Fig. 1), then the coordinates of point $\mathrm{T}$.

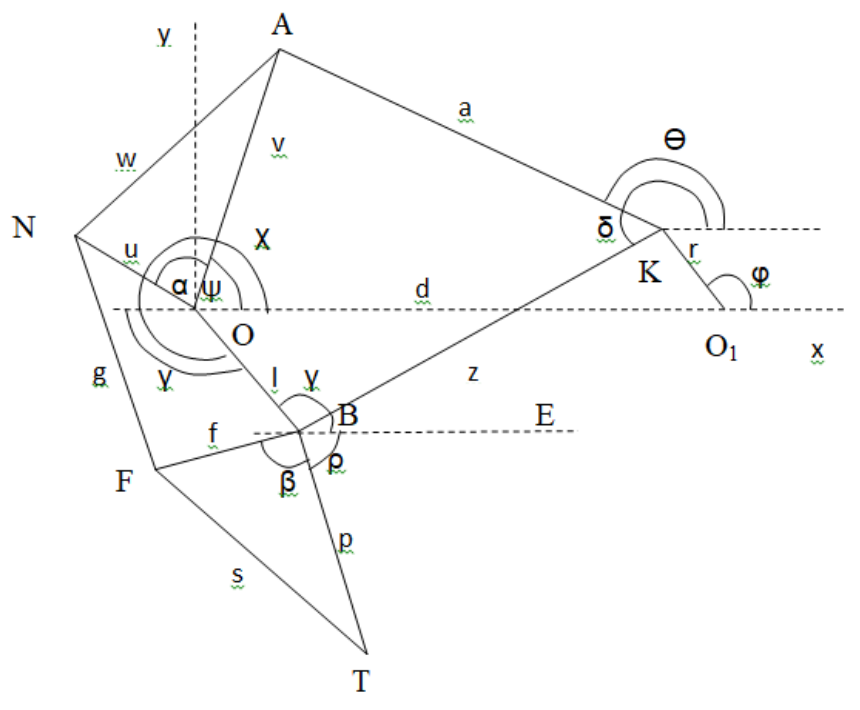

Fig. 1. Scheme of the stepping mechanism.

$\left\{\begin{array}{l}x_{T}=x_{B}+p \cdot \cos \rho \\ y_{T}=y_{B}+p \cdot \sin \rho\end{array}\right.$,

where $x_{B}, y_{B}$ are the coordinates of point $B$, angle $\rho$ is the angle between horizontal BE and side BT of the lower triangle (Fig. 1). To obtain the coordinates of point $\mathrm{B}$, the vector equality is used: 
$\overrightarrow{O B}=\overrightarrow{O K}+\overrightarrow{K B}$,

where $\overrightarrow{O K}=\overrightarrow{O O}_{1}+\overrightarrow{O_{1} K}$.

Or:

$\vec{l}=\vec{d}+\vec{r}+\vec{z}$,

This equality in coordinate form:

$\left\{\begin{array}{l}x_{B}=x_{d}+x_{r}+x_{z} \\ y_{B}=y_{d}+y_{r}+y_{z}\end{array}\right.$
or $\left\{\begin{array}{l}l \cdot \cos \chi=d+r \cdot \cos \phi+z \cdot \cos \delta \\ l \cdot \sin \chi=0+r \cdot \sin \phi+z \cdot \sin \delta\end{array}\right.$,

where $\varphi$ is the angle between the horizontal and $\mathrm{O}_{1} \mathrm{~K}, \chi$ is the angle between $\mathrm{Ox}$ and $\mathrm{OB}, \delta$ is the angle between the horizontal and KB (Fig.1). From (4) it follows:

$z \cdot \sin \delta=l \cdot \sin \chi-r \cdot \sin \phi$

$z \cdot \cos \delta=l \cdot \cos \chi-r \cdot \cos \phi-d$

and by the ratio:

$(z \cdot \sin \delta)^{2}+(z \cdot \cos \delta)^{2}=z^{2}$,

(6) $A^{2}+B^{2}-C^{2} \geq 0$ :

$t_{1,2}=\frac{2 \cdot A \pm \sqrt{4 \cdot A^{2}-4 \cdot(B+C) \cdot(C-B)}}{2 \cdot(B+C)}=\frac{A \pm \sqrt{A^{2}+B^{2}-C^{2}}}{B+C}$,

then

$\chi_{1: 2}=2 \cdot \operatorname{arctg}(t)$.

The graph of $\chi_{2}$ depending on angle $\varphi$ is shown in Fig. 2, and angle $\chi_{1}$ corresponds to an unrealizable trajectory (the mechanism is mirrored relative to the horizontal). 


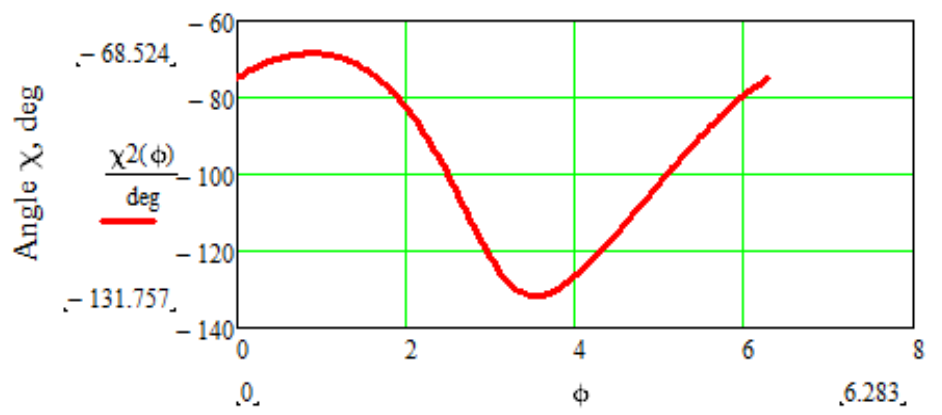

Angle $\varphi$, rad

Fig. 2. Dependence of $\chi_{2}$ on angle $\varphi$.

The coordinates of point B (Fig. 3) will be:

$\left\{\begin{array}{l}x_{B}=l \cdot \cos \left(\chi_{2}(\varphi)\right. \\ y_{B}=l \cdot \sin \left(\chi_{2}(\varphi)\right.\end{array}\right.$.

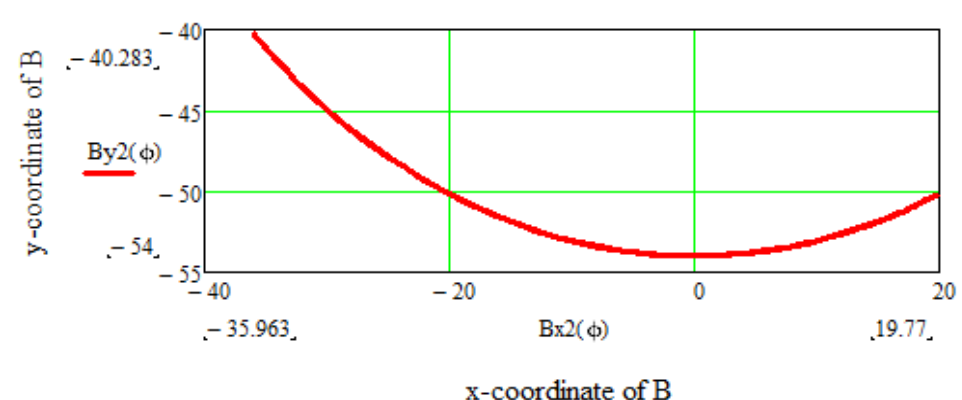

Fig. 3. Trajectory of point.

found:

$\overrightarrow{O A}=\overrightarrow{O K}+\overrightarrow{K A}$.

Taking into account that

$\overrightarrow{O A}=\overrightarrow{O K}+\overrightarrow{K A}$, then

$\vec{v}=\vec{d}+\vec{r}+\vec{a}$.

The coordinate form of these relationships is:

$(v \cdot \sin \psi-r \cdot \sin \varphi)^{2}+(v \cdot \cos \psi-(r \cdot \cos \varphi+d))^{2}=a^{2}$, 
we find $A_{1}$ :

$A_{1} \cdot \sin \psi+B_{1} \cdot \cos \psi=C_{1}$,

where

$A_{1}=2 \cdot v \cdot r \cdot \sin \phi$,

$B_{1}=2 \cdot v \cdot r \cdot \cos \phi+2 \cdot d \cdot v$,

$C_{1}=v^{2}+r^{2}+d^{2}+2 r d \cdot \cos \varphi-a^{2}$,

In B (19) a replacement is used:

$\sin \psi=\frac{2 \cdot \tau}{1+\tau^{2}}, \quad \cos \psi=\frac{1-\tau^{2}}{1+\tau^{2}}$,

where $\psi \neq \pi+2 \pi n \quad$ и $\tau=\operatorname{tg}\left(\frac{\psi}{2}\right)$,

resulting in a quadratic equation in relation to $\tau$ :

$$
\left(B_{1}+C_{1}\right) \cdot \tau^{2}-2 \cdot A_{1} \cdot \tau+\left(C_{1}-B_{1}\right)=0 .
$$

The roots of this quadratic equation:

$\tau_{1,2}=\frac{2 \cdot A_{1} \pm \sqrt{4 \cdot A_{1}^{2}-4 \cdot\left(B_{1}+C_{1}\right) \cdot\left(C_{1}-B_{1}\right)}}{2 \cdot\left(B_{1}+C_{1}\right)}=$

$\frac{A_{1} \pm \sqrt{A_{1}^{2}+B_{1}^{2}-C_{1}^{2}}}{B_{1}+C_{1}}$

with the corresponding angles:

$\psi_{1,2}=2 \cdot \operatorname{arctg}\left(\tau_{1,2}\right)$,

Thus, $\psi_{1,2}$ will be real if the discriminant is positive $A_{1}{ }^{2}+B_{1}{ }^{2}-C_{1}{ }^{2} \geq 0$. The dependence of $\psi_{1}$ on angle $\varphi$ is shown in Fig. 4 , and the angle $\psi_{2}$ corresponds to an unrealizable trajectory.

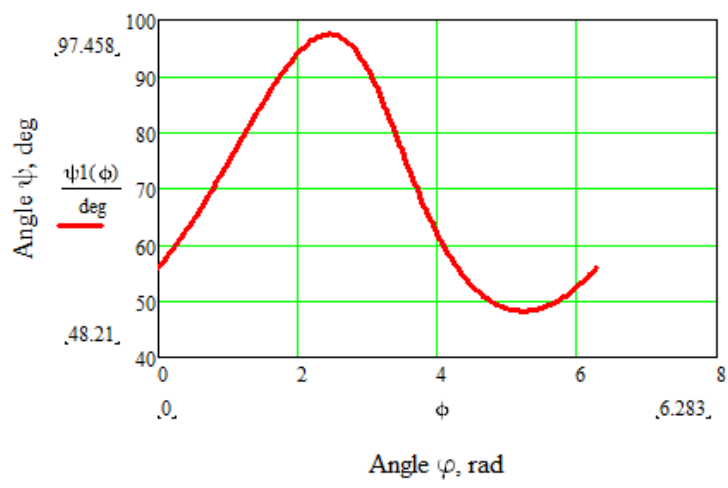

Fig. 4. Dependence of $\psi 1$ on angle $\varphi$.

The coordinates of point A (Fig. 5) are
nd as follows: $\left\{\begin{array}{l}x_{A}=v \cdot \cos \left(\psi_{1}(\varphi)\right. \\ y_{A}=v \cdot \sin \left(\psi_{1}(\varphi)\right.\end{array}\right.$

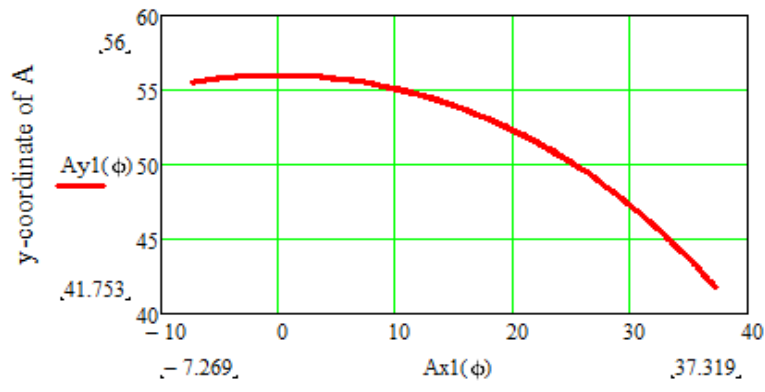

$\mathrm{x}$-coordinate of $\mathrm{A}$

Fig. 5. Trajectory of point A. 
From quadrilateral BFNO (Fig. 6) the angle $\rho$ is determined ( $\mathrm{BE}$ - horizontal):
Angles $v$ and $\eta$ are determined after dividing the quadrilateral by diagonal $b$ into two triangles (Fig. 6).

$$
2 \pi-\rho(\phi)=\eta(\phi)+v(\phi)+\beta+\gamma(\phi) .
$$

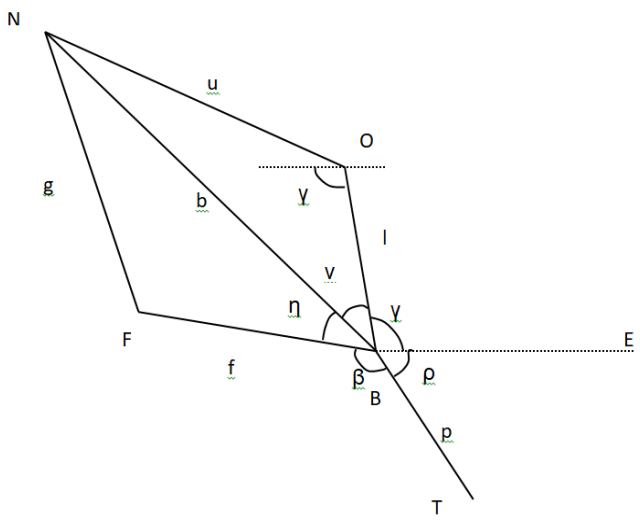

Fig .6. Angles in BFNO (BN=b).

According to the cosine theorem:

$b(\varphi)=\sqrt{u^{2}+l^{2}-2 \cdot u \cdot l \cdot \cos (\gamma(\varphi)}$,

with

$$
\gamma(\varphi)=2 \cdot \pi-\psi_{1}(\varphi)-\chi_{2}(\varphi)-\alpha
$$

where $\alpha$ is found from triangle AON (Fig.1):

$$
\alpha=\arccos \left(\frac{v^{2}+u^{2}-w^{2}}{2 \cdot u \cdot v}\right) .
$$

The change in the length of diagonal $\mathrm{BN}=\mathrm{b}$ depending on angle $\varphi$ is shown in Fig. 7. The graph of Eq. (29) is shown in Fig. 8.

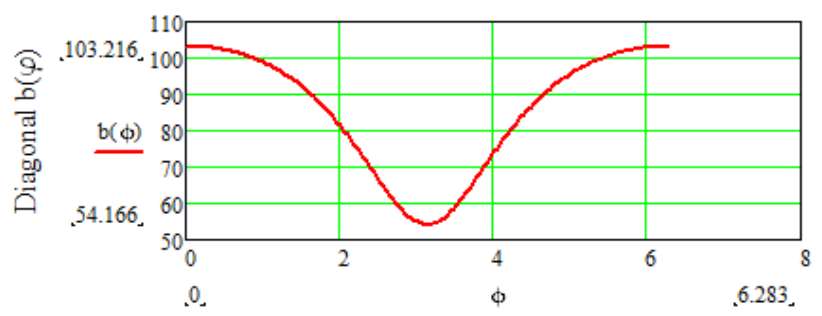

Angle $\varphi$, rad

Fig. 7. Dependence of diagonal b on angle $\varphi$.

Similarly:

$u^{2}=b^{2}+l^{2}-2 \cdot l \cdot b \cdot \cos v$, where 


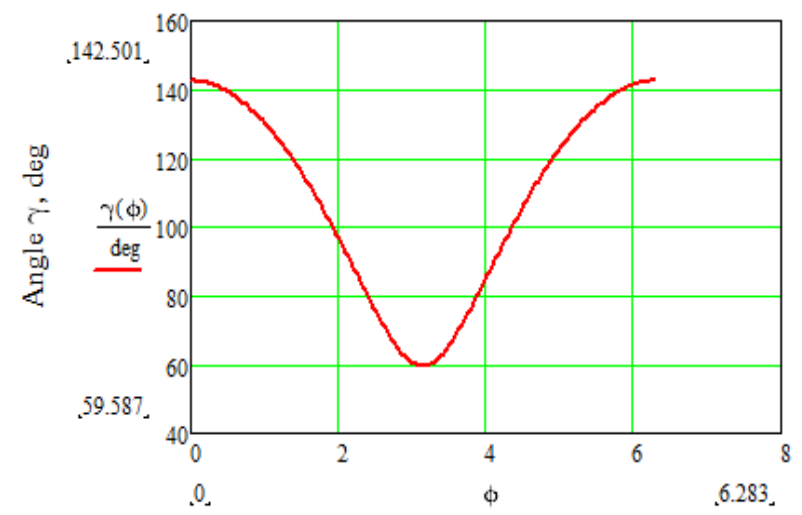

Angle $\varphi$, rad

Fig. 8. Dependence of angle $\gamma$ on $\varphi$.

Similarly:

$g^{2}=b^{2}+f^{2}-2 \cdot b \cdot f \cdot \cos \eta$,

$\eta(\varphi)=\arccos \left(\frac{b(\varphi)^{2}+f^{2}-g^{2}}{2 \cdot b(\varphi) \cdot f}\right)$.

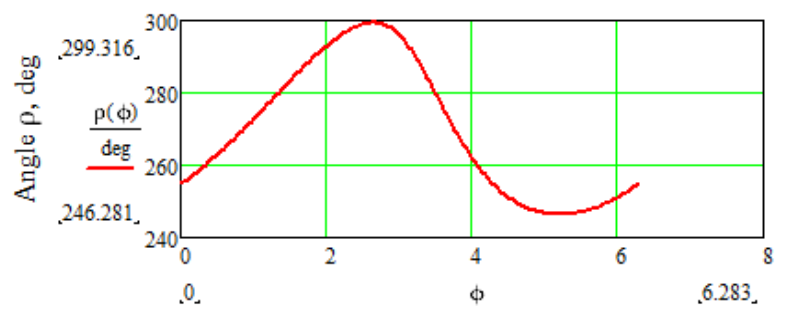

Angle $\varphi$, rad

Fig. 9. Dependence of angle $\rho$ on angle $\varphi$.

Then we find:

$\rho(\varphi)=\pi+\beta+\mathrm{i}(\varphi)+\mathrm{c}(\varphi)-\div(\varphi), \quad(34) \quad s^{2}=f^{2}+p^{2}-2 \cdot f \cdot p \cdot \cos \beta$

where angle $\beta$ is defined from triangle FBT $\quad \beta=\arccos \left(\frac{f^{2}+p^{2}-s^{2}}{2 \cdot f \cdot p}\right)$
(Fig. 1): 
Figures 10-12 show examples of trajectories of stepping point $\mathrm{T}$ defined by (1) for different sizes of the elements of the mechanism.

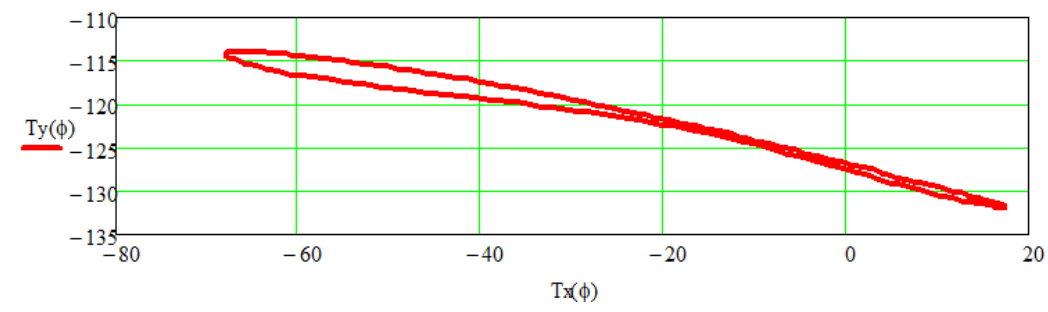

Fig. 10. Invalid trajectory of point $\mathrm{T}$.

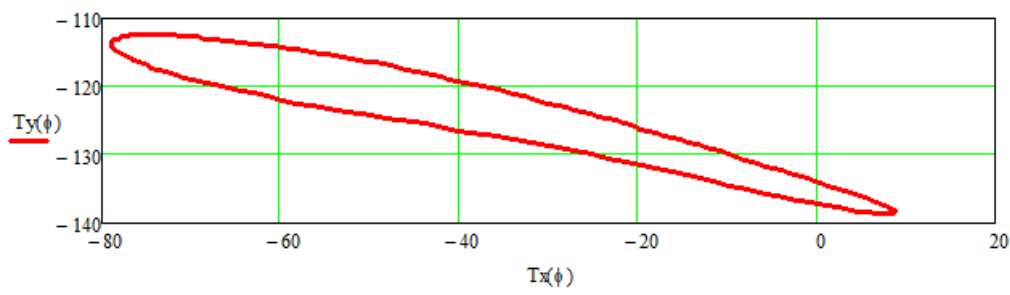

Fig. 11. Acceptable trajectory of point $\mathrm{T}$.

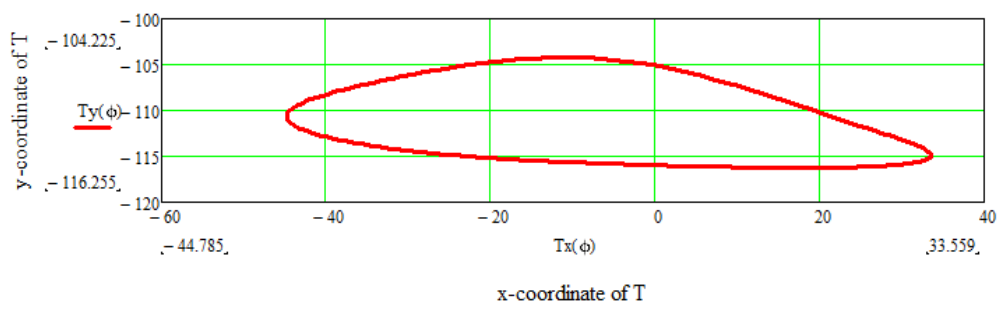

Fig. 12. Optimal trajectory of point $\mathrm{T}$.

\section{RESULTS AND DISCUSSION}

The optimal dimensions of the elements of the stepping mechanism were selected (Fig. 1): $r=20\left(\mathrm{O}_{1} \mathrm{~K}\right), \mathrm{a}=68(\mathrm{KA})$, $\mathrm{z}=85(\mathrm{BK}), \mathrm{d}=61\left(\mathrm{OO}_{1}\right), \mathrm{g}=65(\mathrm{NF}), \mathrm{l}=54(\mathrm{OB})$, $\mathrm{v}=56(\mathrm{OA}), \mathrm{u}=55(\mathrm{NO}), \mathrm{w}=76(\mathrm{AN}), \mathrm{f}=50(\mathrm{FB})$, $\mathrm{p}=66(\mathrm{BT}), \mathrm{s}=90(\mathrm{FT})$. In this case, it is necessary to raise point $\mathrm{O}_{1}$ of the mechanism above the horizontal so that the straight line $\mathrm{OO}_{1}$ makes the angle of $5^{\circ}$ with the horizontal, which brings the lower part of the trajectory (Fig.12) closer to the horizontal. The swing amplitude of the gravity centre of the mechanism in the vertical plane at the given dimensions is approximately 3.2 at the step length of 78.3.

For comparison, Fig. 13 shows the trajectory of the mechanisms created by Theo Jansen, the dimensions of the elements are taken from [4] and are given in the second column of Table 1. Figure 14 shows the tra- 
jectory obtained in [7], the corresponding dimensions of the elements taken from the illustration in this work (the dimensions are not indicated by the authors) and are given in the third column of Table 1 .

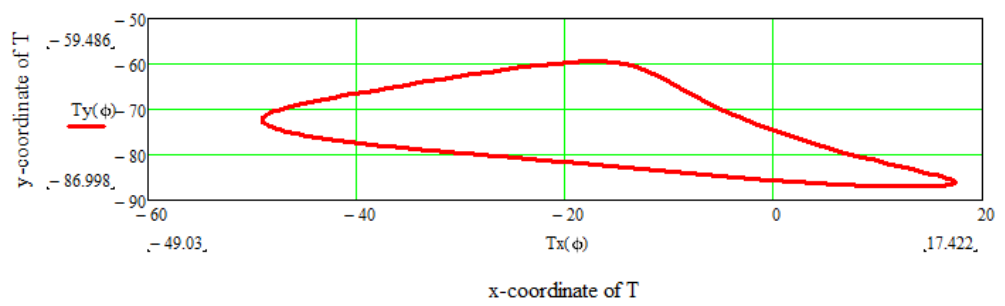

Fig. 13. The trajectory of Theo Jansen's mechanism.

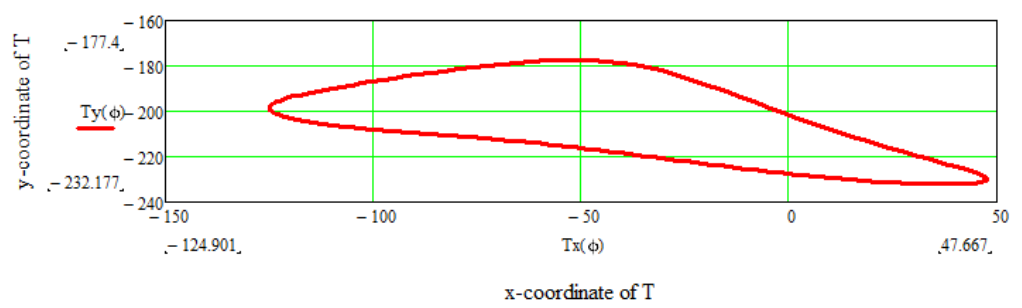

Fig. 14. The trajectory of this work [7].

Table 1. Comparison of Dimensions and Parameters of Mechanisms

\begin{tabular}{|l|c|c|c|}
\hline Parameter & Theo Jansen & Fomin A., et al. [7] & Present research \\
\hline r & 15 & 40 & 20 \\
\hline a & 50 & 137 & 68 \\
\hline z & 61.9 & 167 & 85 \\
\hline d & 38.79 & 107 & 61 \\
\hline g & 39.4 & 106 & 65 \\
\hline l & 39.3 & 107 & 54 \\
\hline v & 41.5 & 111 & 56 \\
\hline u & 40.1 & 108 & 55 \\
\hline w & 55.8 & 149 & 76 \\
\hline f & 36.7 & 97 & 50 \\
\hline p & 49 & 129 & 66 \\
\hline s & 65.7 & 174 & 78.3 \\
\hline Length of the step & 67.8 & 172.5 & 3.2 \\
\hline Swing of occislations & 3.2 & 6.3 & $3.2 / 78.3=0.041$ \\
\hline Ratio & $3.2 / 67.8=0.047$ & $6.3 / 172.5=0.037$ & \\
\hline
\end{tabular}

The bottom line of the table shows the main parameter characterising the oscilla- tions of the centre of masses of the mechanism in the vertical plane, which is equal to 
the ratio of the swing of oscillations to the step length of the mechanism. The trajectory of this work (Fig. 12) in this parameter exceeds the trajectory of Theo Jansen (Fig. 13), but it is less than the trajectory of [7] (Fig. 14). However, approximately in the centre, the lower part of the trajectory of work [7] has a slight deflection upward from the desired straight line, which generates unwanted oscillations of the mechanism with a higher frequency compared to the main frequency of a full step.

\section{CONCLUSIONS}

The trajectories of Theo Jansen and work [7] have a common defect - a sharp shape of the rear part. The transition from the bottom of the trajectories to the top part is not smooth. The trajectory proposed in this paper is free from this drawback and is smooth. These rationales allow us to consider the trajectory proposed in this study as optimal.

\section{REFERENCES}

1. Jansen, B. L., Doubrovski, E., \& Verlinden, J. C. (2014). Animaris Geneticus Parvus: Design of a Complex Multi-Body Walking Mechanism. Rapid Prototyping Journal, 20 (4), 311-319.

2. Jansen, T. (2007). The great pretender. 10 Publishers.

3. Somak, S., \& Pramod, B. (2017). Study of Applications of Jansen's Mechanism in Robot. International Journal of Advance Research and Innovation, 5 (3), 354-357.

4. Ghassaei, A. (2011). The Design and Optimization of a Crank-Based Leg Mechanism. PhD Thesis. Pomona College Department of Physics and Astronomy, Claremont.
5. Lovasz, E. Ch., Pop, C., Pop, F., \& Dolga V. (2014). Novel Solution for Leg Motion with 5-Link Belt Mechanism. International Journal of Applied Mechanics and Engineering, 19 (4), 699-708.

6. Komoda, K., \& Wagatsuma, H. A. (2011). Study of Availability and Extensibility of Theo Jansen Mechanism toward Climbing over Bumps. The 21st Annual Conference of the Japanese Neural Network Society, $3-28$.

7. Fomin, A. S., Kiselev, S. V., \& Oleksenko, A. V. (2017). Strukturnij analiz mehanizma Jansena. Vestnik Sibirskovo gosudarstvennovo industrialnovo universiteta, 4 (22), 51-53. 